\title{
Realization of an atomic sieve: Silica on Mo(112)
}

\author{
Stefan Ulrich, Niklas Nilius ${ }^{*}$, Hans-Joachim Freund \\ Fritz-Haber-Institut der MPG, Berlin, Germany \\ Umberto Martinez, Livia Giordano, Gianfranco Pacchioni \\ Dipartimento di Scienza dei Materiali, Università di Milano-Bicocca, \\ Via R. Cozzi, 53 - 20125, Milano, Italy
}

The adsorption of $\mathrm{Pd}, \mathrm{Ag}$ and $\mathrm{Au}$ atoms on a porous silica film on $\mathrm{Mo}(112)$ is investigated by scanning tunneling microscopy and density functional theory. While Pd atoms are able to penetrate the holes in the silica top-layer with virtually no barrier, Ag atoms experience an intermediate barrier value and Au atoms are completely unable to pass the oxide surface. The penetration probability does not correlate with the effective atom size, but depends on their electronic structure. Whereas Pd with an unoccupied valence s-orbital has a low penetration barrier, Ag and $\mathrm{Au}$ atoms with occupied s-states experience a substantial repulsion with the filled oxide states, leading to a higher barrier for penetration. In the case of Ag, the barrier height can be temporally lowered by promoting the Ag 5s-electron into the support. The Mo-supported silica film can thus be considered as a primitive form of an atomic sieve whose selectivity is controlled by the electronic structure of the adatoms.

\section{Keywords:}

Adsorption, Oxide surfaces, Scanning tunneling microscopy, Density functional theory

*Corresponding author: nilius@fhi-berlin.mpg.de 


\section{INTRODUCTION}

Zeolites and silicates have immense technological importance due to their unique structural properties, for instance as high-area catalysts, ion-exchangers and molecular sieves. ${ }^{1,2,3}$ Such materials obey a universal building principle; an open -Al-Si-O- network encloses an array of nano-pores with rather homogenous diameter. These pores can accommodate single ions, molecules or atomic clusters that might conduct chemical reactions within the three-dimensional (3D) matrix. The efficiency of such processes depends on the geometrical size of the species, which has to match the average pore size of the material. As pore diameters in zeolites and silicates are adjustable between 5-100 $\AA$, size-selective adsorption and reaction schemes can be realized.

Recently, a 2D counterpart for 3D porous materials has been developed. ${ }^{4,5}$ The system consists of an ultra-thin silicon-dioxide film that is prepared on a Mo(112) surface. ${ }^{6,7,8}$ The fundamental building blocks of the oxide layer are six-membered -Si-O- rings, enclosing a hole of 3-4 A diameter. These openings provide access to nano-pores located at the Mo-silica interface, which offer an attractive binding environment for metal atoms and small molecules. ${ }^{9,10}$ The oxide surface, on the other hand, is chemically inert due to the high degree of bond saturation in the topmost silica layer. ${ }^{11}$ The adsorption properties of $\mathrm{SiO}_{2} / \mathrm{Mo}(112)$ therefore depend on the ability of adsorbates to pass the openings in the oxide layer and to reach high-binding sites at the interface. So far, only the penetration of Pd atoms through the silica top-layer could be verified experimentally, ${ }^{10}$ although a similar behavior is predicted for $\mathrm{Li}$ and $\mathrm{Na}^{12}$ In contrast, $\mathrm{Au}$ atoms experience a substantial barrier for penetration and are in fact unable to bind to the defect-free oxide surface. ${ }^{9,10}$ The relevant parameter that governs the energy barrier for passing the holes in the oxide film has not been identified yet for the different atoms. In a first, intuitive picture, it might be connected to the atom size with respect to the pore diameter. In this paper, we will show that this approach is too simple and metal atoms of comparable size have different penetration probabilities.

For this purpose, the binding behavior of $\mathrm{Pd}, \mathrm{Ag}$ and $\mathrm{Au}$ atoms onto $\mathrm{SiO}_{2} / \mathrm{Mo}(112)$ is investigated by scanning tunneling microscopy (STM) and compared to results of density functional theory (DFT) calculations. The three species are chosen, because they either exhibit comparable van der Waals radii (Pd and $\mathrm{Au}$ ) or similar electronic properties ( $\mathrm{Au}$ 
and Ag). The derived binding characteristic demonstrates that the probability for an adatom to pass the holes in the silica top-layer is governed by its electronic structures, in particular by the spatial extension and electron filling of its valence orbitals. In extension to earlier work, ${ }^{10}$ a mechanistic picture for the penetration process is provided and substantiated with the help of three examples.

\section{EXPERIMENT and THEORY}

The experiments are carried out in a custom-build, ultra-high vacuum STM operated at $10 \mathrm{~K}$. Imaging is performed in the constant current mode, whereas the sample electronic properties are deduced from differential conductance (dI/dV) measurements applying lock-in technique. According to the Tersoff-Hamann theory for vacuum tunneling, ${ }^{13}$ the $\mathrm{dI} / \mathrm{dV}$ signal provides a measure for the local density of electronic states (LDOS) in the sample.

The Mo support is cleaned by alternating cycles of $\mathrm{Ar}^{+}$sputtering and annealing to 1800 $\mathrm{K}$ and afterwards exposed to $50 \mathrm{~L} \mathrm{O}_{2}$ to produce an oxygen-induced $\mathrm{p}(2 \times 3)$ precursor

structure. ${ }^{14}$ Deposition of $1.2 \mathrm{ML} \mathrm{Si}$ in $1 \times 10^{-7} \mathrm{mbar}_{2}$ at $800 \mathrm{~K}$ and subsequent heating to $1200 \mathrm{~K}$ leads to the formation of a crystalline silica layer characterized by a sharp c(2×2) LEED pattern. ${ }^{4,8,15}$ STM topographic images clearly reveal the porous structure of the silica film (Fig. 1A). Each hole is surrounded by a hexagonal -Si-O- ring of $5.5 \AA$ diameter that is anchored to the Mo support via oxygen atoms below each Si (see model in Fig.2B). The hole opens into a nano-pore located at the $\mathrm{Mo}^{-\mathrm{SiO}_{2}}$ interface that offers a bridge and a top Mo site for binding adsorbates. The main structural defect in the oxide film are [110]-oriented antiphase-domain boundaries (APDB) that form between two domains shifted by a [111] -oriented Mo unit cell vector (arrows in Fig.1). To bridge the displacement vector, the -Si-O- hexagons are replaced by four and eight-membered rings alternating along the APDB. The central hole in the octagonal rings is hereby two times larger than for the regular hexagons. ${ }^{8}$ Single $\mathrm{Pd}, \mathrm{Ag}$ and $\mathrm{Au}$ atoms are deposited from three different mini-evaporators consisting of the respective high-purity wires wrapped around a tungsten filament. Although atom deposition has been performed at $20 \mathrm{~K}$ sample temperature, the thermal energy of the incoming atoms ensures transient diffusion into their equilibrium adsorption sites. 
Spin-polarized DFT calculations are performed in the VASP code, using the generalized gradient approximation, the PW91 exchange-correlation functional and a plane wave basis set (kinetic energy cut-off $400 \mathrm{eV}$ ). ${ }^{16,17}$ The electron-ion interactions are introduced by the projector augmented wave method. ${ }^{18}$ The regular sites in the silica film are modeled with a $(4 \times 2)$ super-cell containing 7 Mo layers $\left(\mathrm{Mo}_{56} \mathrm{Si}_{8} \mathrm{O}_{20}\right)$, while a $(5 \times 2)$ cell with 4 Mo layers is used for the APDB $\left(\mathrm{Mo}_{40} \mathrm{Si}_{10} \mathrm{O}_{25}\right)$. The slabs are separated by $10 \AA$ of vacuum. Penetration barriers are determined by moving the metal atom along the surface normal into the oxide pore and relaxing the metal/oxide complex for each vertical distance. The computed barriers do not correspond to a real transition state and slightly overestimate the actual barrier height. Topographic STM images are simulated using the Tersoff-Hamann approach. ${ }^{13}$

\section{RESULTS and DISCUSSION}

Figure 1 shows STM topographic images of the silica film after deposition of single Pd, $\mathrm{Ag}$ and $\mathrm{Au}$ atoms (from left to right) for a nominal coverage of $5 \times 10^{12}$ atoms per $\mathrm{cm}^{2}$. The three kinds of ad-species appear with rather different shapes and spatial distributions on the surface, although similar preparation and imaging conditions have been applied in all cases. The Pd-related features are imaged with star-like shapes in a bias window between 0.5-1.5 V. According to earlier studies, this distinct contrast provides evidence for a penetration of Pd atoms through the silica holes and their attachment to a Mo bridge

site at the interface. ${ }^{10}$ In this binding geometry, the $P d 5 s$ orbital hybridizes with the $2 p$ states of the six $\mathrm{O}$ atoms in the hosting -Si-O- ring, thereby increasing the unoccupied LDOS at the oxygen positions. The availability of such Pd-induced states for tunneling is responsible for the star-like appearance of embedded Pd species in the STM images (Fig.2). Only at higher imaging bias (above $2.0 \mathrm{~V}$ ), the $P d 5 s$ orbital becomes directly accessible for electrons from the tip and the contrast localizes at the Pd position in the ring center (not shown). ${ }^{10}$ The embedded Pd atoms are randomly distributed on the silica surface and show no preference for binding to APDB (Fig.1A). From analyzing several STM images, an equal probability is deduced to find Pd in regular -Si-O- hexagons and octagonal rings along the APDB, indicating a similar penetration barrier in both cases. 
After Ag deposition, the same hexangular stars appear on the silica surface as for Pd, although in a slightly different bias regime (Fig. 2). The features are visible at small negative and positive voltages, but transform into round protrusions already above $1.5 \mathrm{~V}$ (Fig.1B). The presence of hexangular stars provides a first indication that also Ag atoms are able to penetrate the holes in the -Si-O- hexagons. In contrast to the Pd species, Ag shows a certain affinity to interact with the APDB. In Fig.1B, one out of 18 atoms occupy a -Si-O- octagon, as marked by the square. The overall fraction of Ag atoms bound to APDB is determined to $5 \%$, which compares to $1-2 \%$ of all available sites being located along the domain boundaries. Consequently, the probability to find an Ag is 3-5 times higher for the -Si-O- octagons than for the hexagons, suggesting a slightly lower penetration barrier at the APDB.

A completely different behavior is revealed for the Au atoms. No adsorbates are found on defect-free silica patches after gold deposition, suggesting that Au atoms are unable to pass the holes in the -Si-O- hexagons and do not bind atop the surface either. Apparently, the penetration barrier for regular -Si-O- rings is too large to be overcome by the thermal $\mathrm{Au}$ atoms, which arrive with $\sim 90 \mathrm{meV}$ according to the gold evaporation temperature. Instead, small Au aggregates line up along the APDB, indicating that the adatoms are able to interact with the eight-membered rings. Fig. 2 shows a close-up STM image of a single $\mathrm{Au}$ atom that has penetrated such an octagonal -Si-O- ring. It is identified by two bright spots emerging at the long sides of the ring opening at low imaging bias. As deduced from DFT calculations, this distinct contrast manifests a structural distortion of the -Si-O- octagon to allocate more space for the Au atom. ${ }^{10}$ The insertion of a single atom into an eight-membered ring already forms the critical nucleus for Au aggregation and initiates the growth of small particles, as seen in Fig. 1C. ${ }^{19}$ In summary, Pd, Ag and Au atoms interact with the silica surface in different ways. While a similar penetration probability of regular -Si-O- hexagons and octagons is found for Pd, the Ag shows a 3-5 times larger probability to pass the eight-membered rings at APDB. In contrast, $\mathrm{Au}$ is completely unable to penetrate the regular silica holes and exclusively binds to boundary sites of the surface.

In the following, the experimental adsorption behavior of the three atomic species is compared to DFT calculations. Pd atoms are found to pass the regular -Si-O- hexagons 
with virtually no barrier (below $50 \mathrm{meV}$ ) and bind with $3.3 \mathrm{eV}$ to interfacial Mo bridge sites (Fig. 3). According to a Bader analysis, the Pd remains neutral during penetration $\left(4 d^{10} 5 s^{0}\right.$ configuration), but becomes partly negatively charged (-0.5e) in its final binding configuration. The penetration and binding energies are similar for the six- and eightmembered rings, explaining the equal distribution of Pd atoms on regular and defect sites of the silica surface. The most pronounced change in the oxide electronic structure upon Pd incorporation is a hybridization between the $P d 5 s$ and $O 2 p$ orbitals of the surface oxygen atoms. The resulting O2p- Pd5s hybrid state is calculated to be at $2.0 \mathrm{eV}$ above the Fermi level and shows up also in the experimental $\mathrm{dI} / \mathrm{dV}$ spectra of Pd atoms as a shoulder at $2.3 \mathrm{~V}$ (Fig. 4). The good agreement between theory and experiment in terms of imaging contrast (Fig.2) and spectroscopic response (Fig.4) verifies the incorporation mechanism of Pd atoms into the silica film.

The $\mathrm{Ag}$ atoms experience a much higher barrier of $0.3 \mathrm{eV}$ for penetrating the sixmembered -Si-O- rings (Fig. 3). Assuming an Arrhenius behavior, thermal Ag atoms have a 30 times lower penetration probability than Pd atoms, which is however still high enough to yield measurable penetration rates. ${ }^{20}$ The Ag atoms are able to overcome this barrier only at the moment of impact. After thermalization, the atoms are trapped in a weak physisorption potential of roughly $0.1 \mathrm{eV}$ and diffuse to adjacent APDB, where penetration of the octagonal rings remains possible. This diffusive transport might be responsible for the higher adsorption probability of Ag atoms at APDB with respect to regular oxide sites. After passing the nano-hole, $\mathrm{Ag}$ binds to the $\mathrm{Mo}-\mathrm{SiO}_{2}$ interface with $1.6 \mathrm{eV}$, remaining in its neutral $4 d^{10} 5 s^{1}$ configuration as above the surface (Fig. 3). The binding energy is substantially lower than for Pd due to the chemically inactive nature of the low-lying $\mathrm{Ag} 4 d$ states. Similar to the Pd case, the largest change in the oxide electronic structure is the formation of an $\mathrm{O} 2 p-\mathrm{Ag} 5 \mathrm{~s}$ hybrid state that peaks at $+1.6 \mathrm{eV}$ and exhibits a long tail crossing the Fermi level (Fig. 4A). Tunneling into this tail produces the typical star-like images of inserted $\mathrm{Ag}$ atoms, originating from the enhanced LDOS of the six $\mathrm{O}$ atoms of the hosting ring (Fig. 2). Above $1.5 \mathrm{~V}$, the $\mathrm{O} 2 p-\mathrm{Ag} 5 \mathrm{~s}$ hybrid state becomes directly available for electron transport and the imaging contrast localizes at the Ag atoms (Fig. 1B). Also the resonance state of inserted Ag shows up experimentally as a $\mathrm{d} / \mathrm{dV}$ peak at $2.05 \mathrm{~V}$, supporting the proposed binding mechanism. 
The calculated penetration barrier of the -Si-O- hexagons increases to $0.9 \mathrm{eV}$ for $\mathrm{Au}$ atoms (Fig. 3). The probability to reach a high binding site at the interface is therefore four orders of magnitude lower than for Pd, explaining the absence of Au species on defect-free silica patches. The penetration of regular $\mathrm{SiO}_{2}$ rings is, however, not only kinetically hindered. Even at higher temperature, desorption and not penetration of $\mathrm{Au}$ atoms would be the dominant process, as the Au binding energy to the silica surface is with $0.1 \mathrm{eV}$ considerably lower than the penetration barrier. Gold is able to pass the oxide surface exclusively at the APDB, where the pore sizes are larger and high-binding interfacial sites are accessible without substantial barrier. Whereas Au atoms are neutral above the silica film ( $5 \mathrm{~d}^{10} 6 \mathrm{~s}^{1}$ configuration), they become partly negatively charged (-0.4 e) at the interface due to an electron transfer from the Mo (Fig. 3). Also from an electronic point of view, Au atoms bound to APDB behave differently than their Pd and Ag counterparts. The Au 6s orbital strongly couples to the Mo states, while hybridization with the oxide LDOS is negligible due to a large energy mismatch (Fig.4A). In contrast to the Pd and Ag-induced states that have substantial density probability even above the oxide surface, the Au 6s-derived states are localized at the Mo-silica interface and therefore inaccessible for $\mathrm{dI} / \mathrm{dV}$ spectroscopy (Fig.4B). In fact, the imaging contrast of $\mathrm{Au}$ atoms bound to APDB results mostly from a structural distortion of the silica lattice upon Au incorporation. ${ }^{10}$

The origin for the dramatically different penetration barriers for Pd, Ag and $\mathrm{Au}$ atoms shall be discussed in the last part of the paper. In a first intuitive picture, the barrier might be connected to the size of the respective gas-phase atoms, as given by their van der Waals radius. ${ }^{21}$ Following this model, smaller atoms should exhibit a reduced barrier with respect to larger ones, a trend that is indeed observed for Pd (van der Waals radius of $163 \mathrm{pm}$ ) versus Ag (172 pm). However, the penetration barrier for Au is substantially higher, although its van der Waals radius of $165 \mathrm{pm}$ is relatively small due to the relativistic contraction of $\mathrm{Au}$ orbitals. The atomic radius seems therefore to be an inappropriate parameter to rationalize the observed trend in barrier height. In a more sophisticated model, the penetration barrier might be linked to the repulsion that is exerted by the oxide charge density, especially the occupied $O 2 p$ states, on the incoming atom. This interaction will be dominated by the spatially most expanded adatom orbital, 
viz the s-like valence states. Due to its unfilled nature, the $P d 5 s$ orbital produces only a small repulsion and the resulting penetration barrier is low. In contrast, the half-filled $\mathrm{Ag}$ $5 s$ and $A u$ 6s orbitals will strongly interact with the surface $O 2 p$ states during penetration. The surprising fact that Ag has a three times lower energy barrier than Au is explained by a transient positive charging of Ag atoms when passing the ring plane. The Ag atom transfers charge density from its $5 \mathrm{~s}$ orbital into the Mo states, thereby reducing the electron-electron interaction with the oxide states. This effect is clearly revealed from the computed Bader charge of $+0.6 e$ for an Ag atom in the ring plane (Fig. 3).

A similar mechanism is not accessible for gold. An Au atom is a strongly electronegative species and rather accumulates excess electrons in its $6 \mathrm{~s}$ orbital instead of transferring charge density to adjacent atoms. ${ }^{22,23}$ Consequently, the Au remains neutral when passing the hole in the silica top-layer (Fig.3). Also an internal charge redistribution, e.g. by promoting the $6 \mathrm{~s}$ electron into the 'leaner' $6 p_{z}$ having its electron density localized along the penetration axis, is inhibited because of the high energy of 4.6 $\mathrm{eV}$ required for this process. ${ }^{24}$ The $\mathrm{Au}$ atoms are therefore unable to lower the repulsive interaction with the oxide surface electrons and the penetration barrier remains high.

\section{CONCLUSION}

The nano-porous $\mathrm{SiO}_{2}$ film grown on $\mathrm{Mo}(112)$ exhibits essential properties of an atomic sieve. Single metal adatoms can either penetrate the openings in the top-most silica layer, followed by a strong binding at the metal-oxide interface, or are repelled at the inert oxide surface. As demonstrated by the combined STM / DFT study, the oxide penetration barrier varies considerably for different atomic species. Whereas Pd atoms experience virtually no barrier, the activation energy for penetration steeply increases when going to $\mathrm{Ag}$ and $\mathrm{Au}$ atoms. The actual barrier height is connected to a repulsive interaction between the valence electrons of the incoming atom and the electron density at the oxide surface, and therefore a purely electronic effect. Having identified this interaction mechanism now enables a reliable prediction of penetration barriers for other atomic and molecular species, being a first step towards the construction of a true atomic sieve based on the $\mathrm{SiO}_{2} / \mathrm{Mo}$ system. 


\section{ACKNOWLEDGMENTS}

The work has been supported by the COST Action D41. Part of the computing time was provided by the Barcelona Supercomputing Center (BSC-CNS).

\section{Figures}
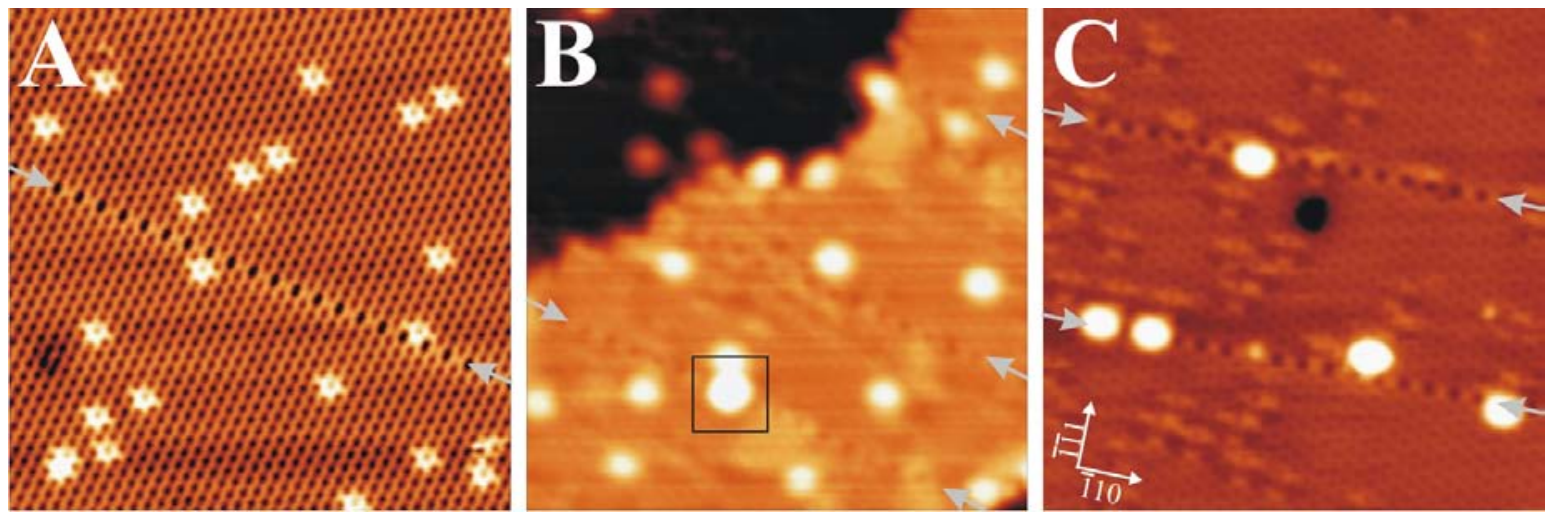

Fig.1

STM topographic images of (A) Pd $\left(\mathrm{U}_{\mathrm{s}}=0.5 \mathrm{~V}\right)$, (B) Ag $\left(\mathrm{U}_{\mathrm{s}}=1.5 \mathrm{~V}\right)$ and $(\mathbf{C}) \mathrm{Au}\left(\mathrm{U}_{\mathrm{s}}=\right.$ $1.0 \mathrm{~V})$ on a thin silica film grown on $\mathrm{Mo}(112)\left(20 \times 20 \mathrm{~nm}^{2}\right)$. The nominal coverage was set to $5 \times 10^{12}$ atoms per $\mathrm{cm}^{2}$ in all cases. The arrows mark domain boundaries in the film. The Ag atom denoted by the square in (B) is bound to such a boundary.
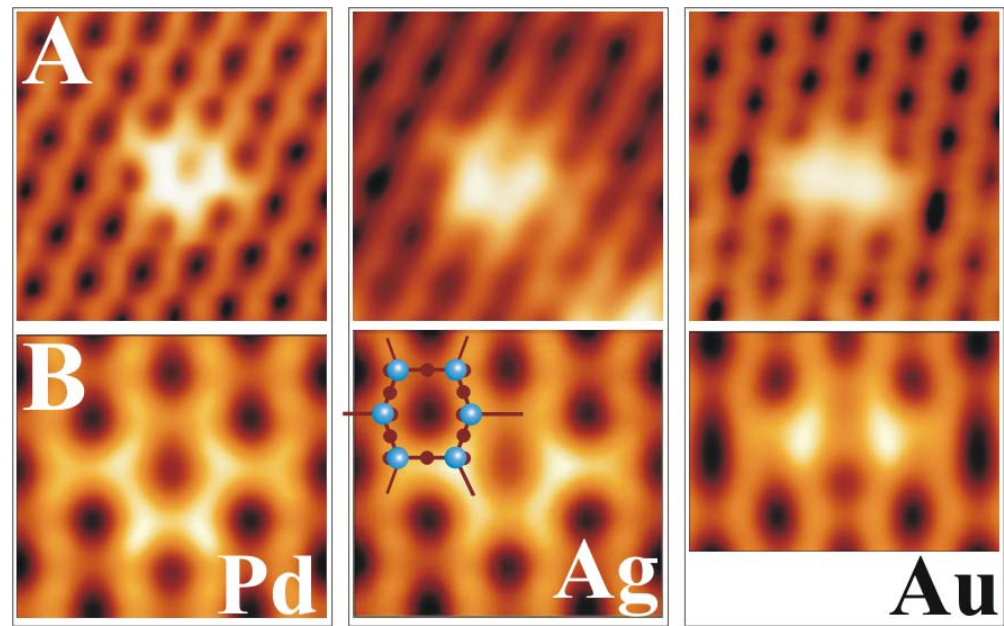

Fig.2

(A) Experimental $\left(5 \times 5 \mathrm{~nm}^{2}\right)$ and (B) simulated STM images (left and center: $1.8 \times 1.6 \mathrm{~nm}^{2}$ and right: $2.1 \times 1.4 \mathrm{~nm}^{2}$ ) of a Pd atom (imaging bias: $\left.0.5 \mathrm{~V}\right)$, an $\mathrm{Ag}(0.3 \mathrm{~V})$ and an $\mathrm{Au}$ atom $(0.8 \mathrm{~V})$ on $\mathrm{SiO}_{2} / \mathrm{Mo}(112)$. While $\mathrm{Pd}$ and $\mathrm{Ag}$ atoms are incorporated into regular six- 
membered rings, the $\mathrm{Au}$ is bound to a $\mathrm{Si}-\mathrm{O}$ octagon located at a domain boundary. The ball model in (B) shows a single -Si-O- hexagon ( $\mathrm{Si}$ : blue, O: red)

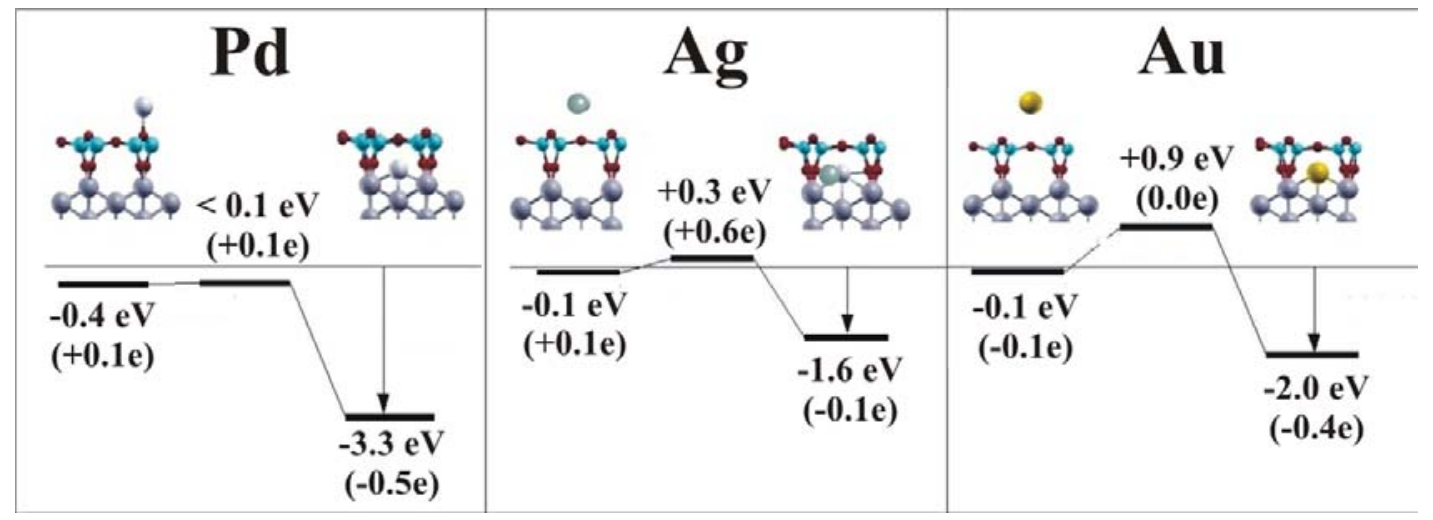

Fig.3

Energy profiles for the insertion of $\mathrm{Pd}, \mathrm{Ag}$ and $\mathrm{Au}$ atoms into regular six-membered rings of the silica film. Left and right values in each plot denote the atom binding energy on top and inside the film, respectively, while the central value gives the energy barrier for penetration. All energies are given with respect to a gas-phase atom. The Bader charges during penetration are reported in parenthesis. The binding configurations of the different atoms above and below the silica film are shown in the insets (Mo: gray, O: red, Si: blue spheres).
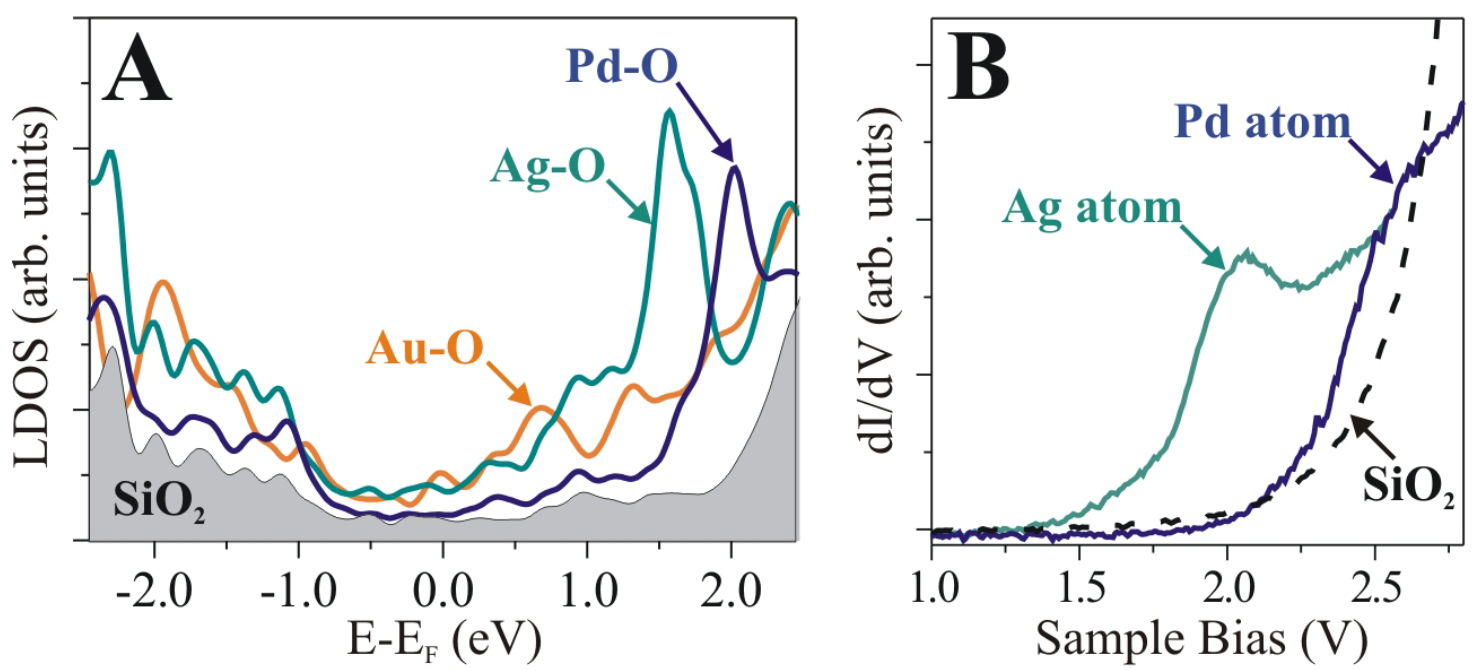

Fig.4

(A) Total LDOS of the pristine silica film (gray) and plots of the hybrid states formed between s-orbitals of incorporated $\mathrm{Pd}, \mathrm{Ag}$ and $\mathrm{Au}$ atoms and the $\mathrm{O} 2 p$ states of the surface oxygen atoms. (B) Experimental dI/dV spectra of the pristine oxide film and inserted Pd and Ag atoms, showing similar resonance states as in (A). 
[1] D. W. Breck, Zeolite molecular sieves: stucture, chemistry, and use, Wiley, New York, 1974.

[2] H. van Bekkum, E. M. Flanigen, J. C. Jansen, [Eds.], Introduction to zeolite science and practice, Elsevier, Amsterdam, 1991.

[3] S. Bhatia, Zeolite catalysis: principles and applications, CRC Press, Boca Raton, 1990.

[4] T. Schroeder, M. Adelt, B. Richter, M. Naschitzki, M. Bäumer, H.-J. Freund, Surf. Rev. \& Lett. 7 (2000) 7.

[5] D. Stacchiola, S. Kaya, J. Weissenrieder, H. Kuhlenbeck, S. Shaikhutdinov, H.-J. Freund, M. Sierka, T. K. Todorova, J. Sauer, Angew. Chem. Int. Ed. 118 (2006) 7798.

[6] Y. D. Kim, T. Wei, D. W. Goodman, Langmuir 19 (2003) 354.

[7] B. K. Min, W. T. Wallace, D. W. Goodman, J. Chem. Phys. B 108 (2004) 14609.

[8] J. Weissenrieder, S. Kaya, J.L. Lu, H. Gao, S. Shaikhutdinov, H.-J. Freund, M. Sierka, T. K. Todorova, J. Sauer, J. Phys. Rev. Lett. 95 (2005) 076103.

[9] L. Giordano, A. Del Vitto, G. Pacchioni, J. Chem. Phys. 124, (2006) 034701.

[10] S. Ulrich, N. Nilius, H-J. Freund, U. Martinez, L. Giordano, G. Pacchioni, ChemPhysChem. 9 (2008) 1367.

[11] J. B. Giorgi, T. Schröder, M. Bäumer, H.-J. Freund, Surf. Sci. Lett. 498 (2002) L71.

[12] U. Martinez, L. Giordano, G. Pacchioni, J. Chem. Phys. 128 (2008) 164707.

[13] J. Tersoff, D. R. Hamann, Phys. Rev. Lett. 50 (1983) 1998.

[14] T. Schröder, J. B. Giorgi, A. Hammoudeh, N. Magg, M. Bäumer, H.-J. Freund, Phys. Rev. B 65 (2002) 115411.

[15] L. Giordano, D. Ricci, G. Pacchioni, P. Ugliengo, Surf. Sci. 584 (2005) 225.

[16] J. P. Perdew, J. A. Chevary, S. H. Vosko, K. A. Jackson, M. R. Pederson, D. J. Singh, C. Fiolhais, Phys. Rev. B 46 (1992) 6671.

[17] G. Kresse, J. Hafner, Phys. Rev. B 47 (1993) 558; G. Kresse, J. Furthmüller, Phys. Rev. B 54 (1996) 11169.

[18] P. E. Blöchl, Phys. Rev. B 50 (1994) 17953.

[19] K. Min, W. T. Wallace, A. K. Santra, D. W. Goodman, J. Chem. Phys. B 108 (2004) 16339.

[20] Quantitative values for the penetration rate cannot be given as the pre-factor for the Arrhenius process is unknown.

[21] D. R. Lide (Ed.), Handbook of chemistry and physics, CRC Press, Boca Raton, 2008.

[22] G. Pacchioni, L. Giordano, M. Baistrocchi, Phys. Rev. Lett. 94 (2005) 226104.

[23] N. Nilius, V. Ganduglia-Pirovano, V. Bradzova, M. Kulawik, J. Sauer, H.-J. Freund, Phys. Rev. Lett. 100 (2008) 096802.

[24] C. E. Moore (Ed.), Atomic Energy Levels, US-Gov. Print Office, Washington, 1952. 